\title{
The Keck OSIRIS Nearby AGN (KONA) Survey: AGN Fueling and Feedback
}

\author{
Erin K. S. Hicks ${ }^{1}$, Francisco Müller-Sánchez ${ }^{2}$, Matthew A. Malkan ${ }^{3}$ \\ and Po-Chieh $\mathrm{Yu}^{3}$ \\ ${ }^{1}$ University of Alaska Anchorage \\ email: ehicks@uaa.alaska.edu \\ ${ }^{2}$ University of Colorado, Boulder ${ }^{3} \mathrm{UCLA}$
}

Keywords. galaxies: active, galaxies: Seyfert, galaxies: evolution, galaxies: nuclei, surveys

In an effort to better constrain the relevant physical processes dictating the co-evolution of supermassive black holes and the galaxies in which they reside we turn to local Seyfert AGN. It is only with these local AGN that we can reach the spatial resolution needed to adequately characterize the inflow and outflow mechanisms thought to be the driving forces in establishing the relationship between black holes and their host galaxies at higher redshift. We present the first results from the KONA (Keck OSIRIS Nearby AGN) survey, which takes advantage of the integral field unit OSIRIS plus laser and natural guide star adaptive optics to probe down to scales of 5-30 parsecs in a sample of 40 local Seyfert galaxies. With these $K$-band data we measure the two-dimensional distribution and kinematics of the nuclear stars, molecular gas, and ionized gas within the central few hundred parsecs. The stellar kinematics, measured by the $\mathrm{CO}$ bandheads at $2.3 \mu \mathrm{m}$, indicate that the stellar population in this region is in circular rotation. In the majority of the galaxies molecular gas, traced by 1-0 $\mathrm{S}(1) \mathrm{H}_{2}$ emission at 2.12 $\mu \mathrm{m}$, is also in rotation and is co-spatial with the stellar disk. A significant fraction of the Seyferts in the sample also exhibit kinematic signatures of inflow (e.g. inflow along a nuclear spiral; see Fig. 1) as well as outflow in the molecular gas that is superimposed on this disk rotation. Ionized gas detected via $\mathrm{Br} \gamma$ recombination at $2.16 \mu \mathrm{m}$ and [Si VI] emission at $1.96 \mu \mathrm{m}$ in most of the Seyferts shows evidence of outflows, and, in many cases, is interacting with the interstellar medium traced by the molecular gas. The survey sample is divided evenly between type 1 and type 2 Seyferts, which enables a statistically significant comparison of the nuclear stellar and gas properties in the two subsamples. Differences in the prevalence of the primary AGN fueling mechanisms inferred from the gas kinematics in the two subsamples, as well as trends of the nuclear properties with AGN luminosity, are also investigated.
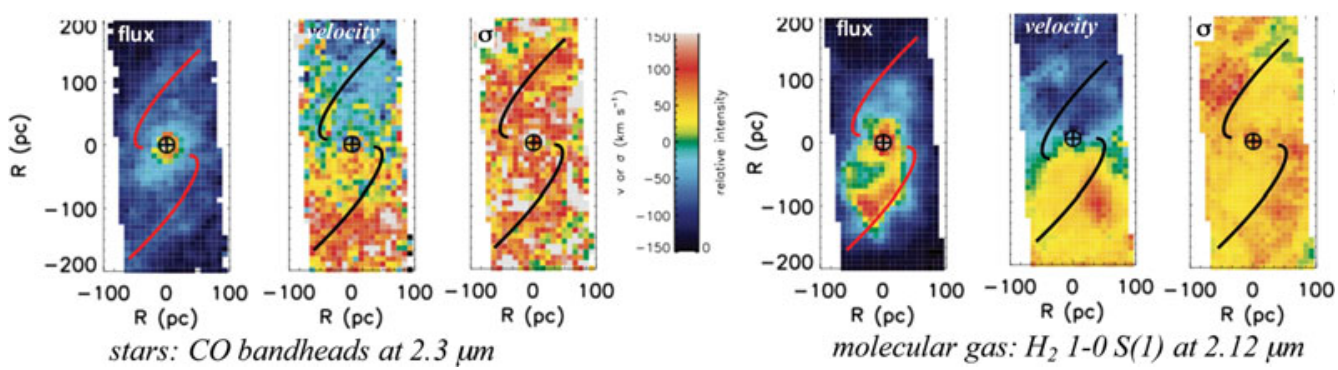

Figure 1. Example maps of the observed flux, velocity, and velocity dispersion (left to right) of the stars (CO absorption) and molecular gas ( $\mathrm{H}_{2}$ emission) for the Seyfert 2 galaxy Mrk 1066 . The red and black lines denote the observed spiral arm structures in the stellar flux distribution, signatures of which can also be seen in the observed flux and kinematics of the gas. 